Notre Dame Journal of Formal Logic Volume 25, Number 4, October 1984

\title{
An Axiomatization of the Equivalential Fragment of the Three-Valued Logic of Łukasiewicz
}

\author{
JACEK K. KABZIŃSKI
}

The problem of axiomatizing the purely equivalential fragment of the infinite-valued Kukasiewicz logic $\left(L_{\infty}\right)$ and the corresponding variety of algebras remains open. Moreover for every $n=3,4, \ldots$ one may ask about axiomatization of the purely equivalential fragment of $n$-valued Kukasiewicz logic $\left(L_{n}\right)$. In this paper we give an axiomatization of the purely equivalential fragment of $L_{3}$ and an appropriate set of identities determining the corresponding variety of algebras (see [3]).

Let us recall that the three-valued logic of Łukasiewicz $L_{3}$ is determined by the following matrix: $L_{3}=\left(\{0,1,2\},\{0\}, \rightarrow_{L}, \wedge_{L}, \vee_{L}, \sim_{L}\right)$ where $x \rightarrow_{L} y=$ $\max (0, y-x), x \wedge_{L} y=\max (x, y), x \vee_{L} y=\min (x, y)$, and $\sim_{L} x=x \rightarrow_{L} 2$ (see [5]).

The other well-known three-valued logic is the logic $H_{3}$ considered by Heyting in [1]. It is determined by the matrix $H_{3}=\left(\{0,1,2\},\{0\}, \rightarrow_{H}\right.$, $\wedge_{H}, \vee_{H}, \sim_{H}$ ), where $x \rightarrow_{H} y=y$ whenever $x<y$ and $x \rightarrow_{H} y=0$ otherwise, $x \wedge_{H} y=\max (x, y), x \vee_{H} y=\min (x, y)$, and $\sim_{H} x=x \rightarrow_{H} 2$.

Let the symbols $L_{3} \equiv$ and $H_{3} \equiv$ denote the purely equivalential fragments in question. Since $x \equiv y=_{d f}(x \rightarrow y) \wedge(y \rightarrow x)$ then $L_{3} \equiv$ and $H_{3}$ are determined by the following matrices $\mathbf{L}_{3} \equiv$ and $\mathbf{H}_{3} \equiv$ respectively: $\mathbf{L}_{3} \equiv=\left(\{0,1,2\},\{0\}, \equiv_{L}\right)$ where $x \equiv_{L} y=\max (x-y, y-x)$ and $\mathbf{H}_{3}=\left(\{0,1,2\},\{0\}, \equiv_{H}\right)$ where $x \equiv_{H} y=$ $\max (x, y)$ whenever $x \neq y$ and $x \equiv_{H} y=0$ otherwise.

It is known that neither $L_{3} \nsubseteq H_{3}$ nor $H_{3} \nsubseteq L_{3}$; for example $(\alpha \rightarrow(\alpha \rightarrow \beta)) \rightarrow$ $(\alpha \rightarrow \beta) \in H_{3}-L_{3}$ whereas $((\alpha \rightarrow \beta) \rightarrow \beta) \rightarrow((\beta \rightarrow \alpha) \rightarrow \alpha) \in L_{3}-H_{3}$. Nevertheless we shall prove that the purely equivalential fragments of $L_{2}$ and $H_{3}$ are identical.

The equality $L_{3} \equiv H_{3}$ is an immediate consequence of the fact that the matrices $\mathbf{L}_{3} \equiv$ and $\mathbf{H}_{3} \equiv$ are isomorphic. The reader will have no difficulty in verifying that the required isomorphism is the mapping $i:\{0,1,2\} \rightarrow\{0,1,2\}$, such that $i(0)=0, i(1)=2, i(2)=1$. 
The notion of intuitionistic equivalential algebra introduced in [2] (see also [4]) is an algebraic counterpart of the equivalential fragment of the intuitionistic propositional logic. This fragment was axiomatized by Tax in [6] by means of the single axiom

(TA)

$$
\begin{aligned}
& ((\beta \equiv(\beta \equiv \alpha)) \equiv((\beta \equiv(\beta \equiv \alpha)) \equiv(\alpha \equiv(\alpha \equiv(\gamma \equiv \delta))))) \\
& \quad \equiv((\alpha \equiv \delta) \equiv(\gamma \equiv \alpha))
\end{aligned}
$$

and the following rules of inference:

(DR) $\frac{\alpha \equiv \beta, \alpha}{\beta} \quad$ (the detachment rule for the equivalence)

(TR) $\frac{\alpha}{\beta \equiv(\beta \equiv \alpha)} \quad$ (the Tax rule).

The class of intuitionistic equivalential algebras was defined in [2] as the variety of all algebras of type $\langle 2\rangle$ satisfying the following identities:

(i1) $(a \equiv a) \equiv b=b$

(i2) $((a \equiv b) \equiv c) \equiv c=(a \equiv c) \equiv(b \equiv c)$

(i3) $((a \equiv b) \equiv((a \equiv c) \equiv c)) \equiv((a \equiv c) \equiv c)=a \equiv b$.

The variety of algebras corresponding to the equivalential fragment of the logic $\mathrm{H}_{3}$ has already been axiomatized in [4] by the identities (i1), (i2), (i3), and

(h1) $\quad(((a \equiv((b \equiv c) \equiv c)) \equiv((b \equiv c) \equiv c)) \equiv((a \equiv((c \equiv b) \equiv b)) \equiv$ $((c \equiv b) \equiv b))) \equiv((a \equiv(b \equiv c)) \equiv(b \equiv c))=a$

(h2) $(a \equiv(((b \equiv c) \equiv c) \equiv b)) \equiv(((b \equiv c) \equiv c) \equiv b)=a$.

On the basis of axioms of the variety of intuitionistic equivalential algebras the identities (h1), (h2) are equivalent to the one identity

(h) $(a \equiv(b \equiv c)) \equiv(b \equiv c)=(a \equiv((a \equiv b) \equiv b)) \equiv((a \equiv c) \equiv c)$.

A routine proof will be omitted.

Since just the same variety corresponds to the equivalential fragment of the logic $L_{3}$, one gets the following:

Corollary The identities (i1), (i2), (i3), (h) form an axiomatization of the variety determined by $L_{3} \equiv$.

The results of Tax [6] combined with our corollary yield the following:

Theorem $L_{3} \equiv$ can be axiomatized by adopting (TA), (DR), (TR), and the following axiom: $((\alpha \equiv(\beta \equiv \gamma)) \equiv(\beta \equiv \gamma)) \equiv((\alpha \equiv((\alpha \equiv \beta) \equiv \beta)) \equiv$ $((\alpha \equiv \gamma) \equiv \gamma))$.

\section{REFERENCES}

[1] Heyting, A., "Die Formalen Regeln der intuitionistischen Logik," Sitzungsberichte der Preussischen Akademie der Wissenschaften, Physikalisch-Mathematische Klasse (1930), pp. 42-56. 
[2] Kabziński, J. K., Algebry Równoważnościowe, Ph.D. dissertation, Jagiellonian University, Krakow, 1974.

[3] Kabziński, J. K., "On equivalential fragment of the three-valued logic of Łukasiewicz" (abstract), Bulletin of the Section of Logic, Polish Academy of Sciences, vol. 8 (1979), pp. 182-187.

[4] Kabziński, J. K. and A. Wroński, "On equivalential algebras," Proceedings of the 1975 International Symposium of Multiple-Valued Logics, Indiana University, Bloomington, May 13-16 (1975), pp. 419-428.

[5] Łukasiewicz, J., "O logice trójwartościowej," Ruch Filozoficzny, vol. 5 (1920), pp. 170-171.

[6] Tax, R. E., "On the intuitionistic equivalential calculus," Notre Dame Journal of Formal Logic, vol. 14 (1973), pp. 448-456.

Department of Logic

Jagiellonian University

31-044 Krakow, Poland 